\title{
LITIASIS RENAL INDUCIDA POR INDINAVIR
}

\author{
Mª L. TRABA VILLAMEYTIDE*, M. FERNÁNDEZ-GUERRERO \\ *Laboratorio de Bioquímica. Sección de Fisiopatología Ósea. Medicina Interna. \\ División de Enfermedades Infecciosas. Fundación Jiménez Díaz. Madrid.
}

Actas Urol Esp. 28 (7): 523-526, 2004

\section{RESUMEN}

LITIASIS RENAL INDUCIDA POR INDINAVIR

$\mathrm{El}$ indinavir es un nuevo, específico y potente fármaco que actúa, como otros agentes anti-retrovirales, inhibiendo la proteasa del virus de la inmunodeficiencia humana (VIH-1) o de la inmunodeficiencia adquirida (SIDA). El indinavir se une al centro activo del enzima, originando un descenso en plasma de ARN VIH-1 y un aumento de los linfocitos T-CD4 "helper" dando origen a un descenso del enzima, necesaria para la maduración y replicación del VIH-1.

El presente trabajo estudia la cristaluria de dos de los nueve pacientes que padecían VIH-1 y/o SIDA tratados con indinavir, así como el cálculo formado por uno de los dos pacientes que presentaban cristaluria.

El estudio se realizó con microscopio de luz polarizada y por espectrofotometría infrarroja, mostrando que la visualización de la cristaluria con microscopio de luz polarizada es útil para la caracterización de las mismas, así como para el estudio de los cálculos renales es útil el análisis por espectrofotometría infrarroja.

PALABRAS CLAVE: Indinavir. VIH-1. SIDA. Cristaluria. Cálculo renal.

\section{ABSTRACT \\ INDINAVIR AND RENAL LITHIASIS}

Indinavir is a new specific and potent drug that inhibits, like other antiretroviral agents, the protease of immune deficiency virus (HIV) or acquired immune deficiency syndrome (AIDS), an enzyme necessary to maduration and replication of the virus. Indinavir has the capacity to bind the active site causing a decrease in plasma of HIV1-RNA and an increase of $\mathrm{T}-\mathrm{CD}_{4}$ helper lymphocytes. The aim of this work is to study in HIV and/or AIDS patients treated with indinavir the crystalluria and the formation of renal calculi due to the clearance of this drug. Two out of nine patients studied in this work presented abundant crystalluria and one of them presented spontaneously passed renal stone.

Urinary crystals were studied under polarized-light microscopy and renal stone was analyzed by infrared spectroscopy.

KEY WORDS: Indinavir. HIV-1. AIDS. Crystalluria. Renal calculi.

$\mathrm{L}^{2}$ a nefrolitiasis es una complicación frecuente bien conocida asociada al tratamiento con indinavir y otros anti-retrovirales en la patología del virus de la inmunodeficiencia humana (VIH1), así como en el síndrome de inmunodeficiencia adquirida (SIDA) ${ }^{1-5}$. La causa de este hecho es debido a que el 20\% del indinavir administrado al paciente es aclarado por el riñón ${ }^{6-9}$. Entre los síntomas producidos por el indinavir hay que destacar la cristaluria ${ }^{3,9}$ y la formación de cálculos renales $^{2,3,7,9-11}$, hechos originados por la poca solubilidad del indinavir en soluciones acuosas. Por eso, el principal factor de riesgo tiene lugar por una ingesta baja en liquidos ${ }^{6}$. El sulfato de 
indinavir es un nuevo y específico fármaco que actúa, como otros agentes anti-retrovirales, inhibiendo la proteasa del VIH-1 o del AIDS, observándose una buena respuesta clínica al producir un descenso en plasma del ARN del VIH-1 y el consiguiente aumento de los linfocitos T-CD4 "helper" ${ }^{13}$, produciendo finalmente un descenso del enzima necesaria para la maduración y replicación del VIH-1 ${ }^{8}$. El modo de acción del indinavir (una hidroxiamino pentano amida ${ }^{8}$ es la unión del fármaco al centro activo del enzi$\mathrm{ma}^{2,8,12}$ con el consiguiente descenso del ARN VIH- $1^{13}$.

Todos los inhibidores de proteasas se metabolizan en el hígado a través del sistema enzimático del citocromo P450 (específicamente del isoenzima $\mathrm{CYP} \mathrm{A} 4^{8}$ ) paso predominante en el metabolismo de los inhibidores de proteasas ${ }^{4,8}$.

$\mathrm{El}$ propósito de este trabajo es analizar la cristaluria que presentan en 2 de los 9 pacientes que padecían VIH-1 y/o SIDA tratados con indinavir, así como el cálculo formado por uno de los dos pacientes que mostraban cristaluria.

\section{MATERIAL Y MÉTODOS}

Durante un periodo de tres meses (agostooctubre de 1998) se estudiaron nueve pacientes que padecían VIH-1 y/o SIDA, tratados con una terapia anti-retroviral con indinavir más una combinación con lamivudine y starvudine. Antes de iniciar el estudio, estos pacientes fueron tratados con indinavir durante un periodo de tiempo de tres a seis meses.

La dosis de indinavir fue de $800 \mathrm{mg}$ cada 8 horas. De los nueve pacientes tratados con indinavir, dos padecieron cólico renal y presentaron abundante cristaluria y uno de ellos expulsó un cálculo renal. Los siete enfermos restantes permanecieron asintomáticos.

Los pacientes recogieron orina de $24 \mathrm{~h}$. A partir de ella se tomaron dos alícuotas que se centrifugaron a $2500 \mathrm{rpm}$ durante 15 minutos. Una de las alícuotas se mantuvo en estufa a $70^{\circ} \mathrm{C}$ durante $4 \mathrm{~h}$. Ambos sedimentos, el tratado y el no tratado con calor, se analizaron con un microscopio con luz polarizada y a ambos también se les realizó un estudio por espectrofotometría infrarroja, por el sistema de comprimidos de bromuro potásico (Espectrofotómetro Perkin Elmer 475).
El cálculo eliminado de forma espontánea por uno de los pacientes fue analizado por espectrofotometría infrarroja, por medio del sistema de comprimidos de bromuro potásico. Un fragmento del cálculo fue sometido en estufa a $70^{\circ} \mathrm{C}$ durante $4 \mathrm{~h}$ y se analizó también por espectrofotometría infrarroja.

Los cambios por espectrofotometría infrarroja, tanto del sedimento como del cálculo, fueron comparados con el espectro realizado a partir del sulfato de indinavir puro (cedido por el Dr. Jorge González Esteban. Merck Sharp and Dohme de España, S.A.).

\section{RESULTADOS}

Dos de los 9 enfermos tratados con indinavir presentaron la típica cristaluria, así como el característico color amarillo de la orina. La cristaluria fue visualizada en microscopio con luz polarizada encontrándose cristales rectangulares o agregados de los mismos en forma de abanico que presentaron birrefringencia con los filtros de polarización cruzados (Fig. 1).

La Figura 2 muestra el espectro de infrarrojos correspondiente al indinavir y a la cristaluria. Las flechas indican las bandas comunes del fármaco con la cristaluria. Aunque no se muestra en el trabajo, no encontramos diferencias en el espectro de infrarrojo de la cristaluria tratada a $70^{\circ} \mathrm{C}$ con respecto a la cristaluria sin tratar por calor.

La Figura 3 muestra el aspecto morfológico del cálculo renal procedente de uno de los enfermos tratados con indinavir.

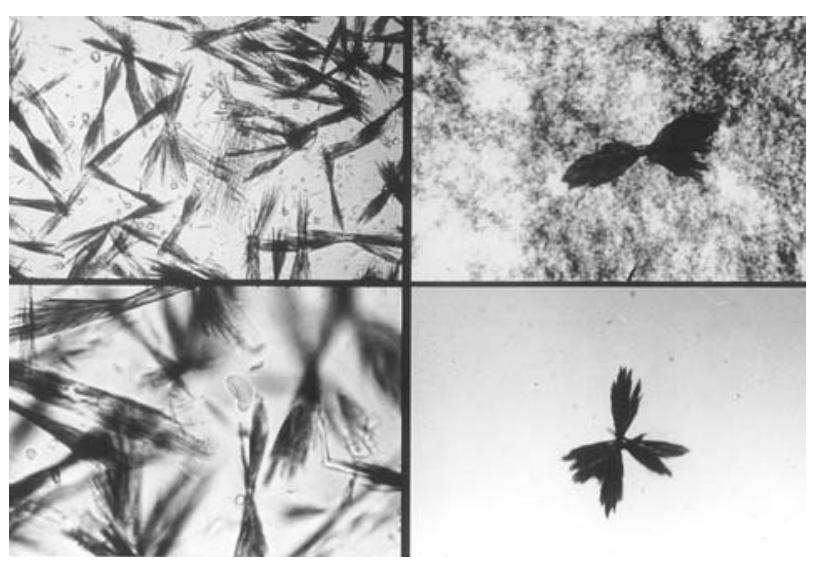

FIGURA 1. Cristaluria procedente de pacientes que padecieron VIH-1 y/o SIDA tratados con indinavir. 


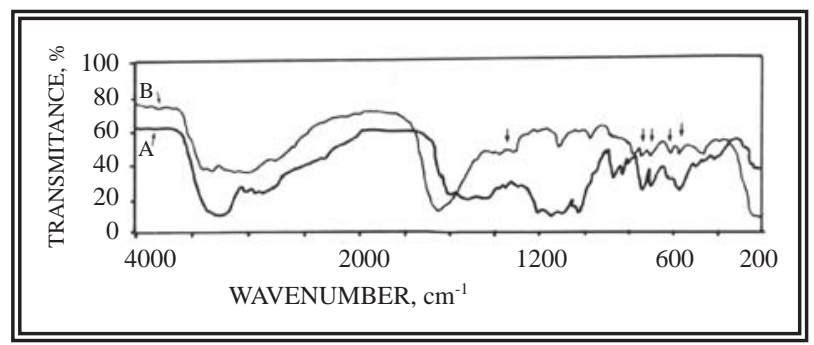

FIGURA 2. Espectro de infrarrojos procedente de pacientes con VIH-1 y/o SIDA tratados con indinavir. A: indinavir puro: B: sedimento.

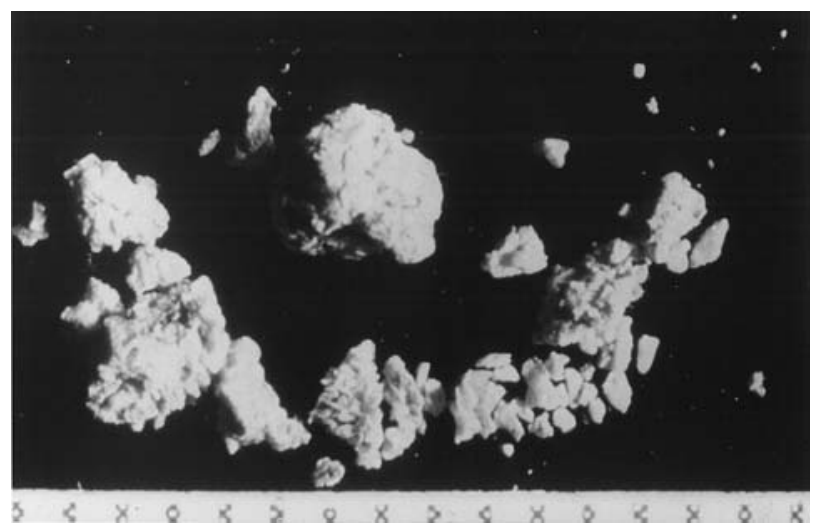

FIGURA 3. Aspecto morfológico del cálculo renal procedente de un enfermo tratado con indinavir puro.

La Figura 4 presenta el espectro de infrarrojos del cálculo y del indinavir. Las flechas indican las zonas del espectro comunes a ambos. Al igual que la cristaluria, no encontramos diferencias entre el espectro de infrarrojos del cálculo tratado con calor a $70^{\circ} \mathrm{C}$ del no-tratado.

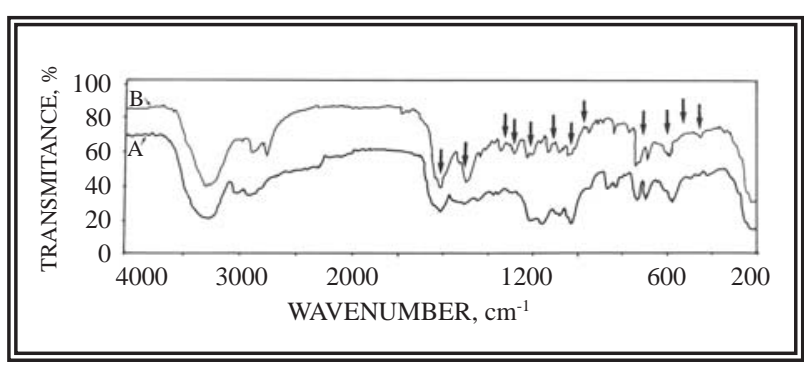

FIGURA 4. Espectro de infrarrojos correspondiente a indinavir puro (A) y al cálculo (B).

\section{DISCUSIÓN}

En los últimos años se ha estudiado la incidencia de los cálculos renales compuestos total o parcialmente por fármacos. Diferentes factores locales de riesgo están implicados en la formación de este tipo de cálculos. Así, una elevada excreción del fármaco y/o de sus metabolitos, una baja solubilidad del mismo, cambios en el volumen de orina, en el $\mathrm{pH}$, así como la duración del tratamiento, puede jugar un papel fundamental en el incremento de saturación del fármaco en la orina o pueden producir un descenso de los factores locales que inhiben la formación de cálculos. Otros factores de riesgo pueden estar implicados en el desarrollo de cálculos renales, como un fracaso renal, enfermedades hepáticas y/o gastrointestinales, así como historia familiar de litiasis ${ }^{10,14}$.

La nefrolitiasis ${ }^{6}$ es uno de los efectos colaterales asociado al tratamiento con indinavir ${ }^{13}$ ya que el $20 \%$ de este fármaco ${ }^{6,9}$ es aclarado por el riñón, bien como componente intacto o en una menor proporción como metabolitos del mismo.

Entre los sintomas producidos por el indinavir hay que destacar la cristaluria y la formación de cálculos renales, como consecuencia de la escasa solubilidad del indinavir en soluciones acuosas.

Perazella y cols. ${ }^{5}$ han encontrado que la solubilidad del indinavir está intimamente ligada a los valores de $\mathrm{pH}$. A pH 7.0 la solubilidad es menor $(0,02 \mathrm{mg} / \mathrm{ml})$ que a $\mathrm{pH} 6.0(0,035 \mathrm{mg} / \mathrm{ml})$ y a $\mathrm{pH}$ 5.0 (alrededor de $0,3 \mathrm{mg} / \mathrm{ml}$ ). Por ello es tan importante la ingesta de líquidos ${ }^{6}$ y que el $\mathrm{pH}$ de la orina sea inferior a $5^{9}$ en los pacientes tratados con indinavir. Salter y cols. ${ }^{10}$ mostraron que el indinavir es un fármaco litogénico que causa cálculos en un 3\% de los enfermos tratados, mientras que Kopp y cols. ${ }^{3}$ encontraron que un $8 \%$ de los enfermos tratados con este fármaco desarrollaron sintomas urológicos y un 3\% padecieron nefrolitiasis. El tratamiento con indinavir según diferentes autores ${ }^{4,5}$ causa nefrolitiasis en un porcentaje variable entre un 3 y un $50 \%{ }^{5}$.

Se han empleado diferentes métodos para el estudio tanto de los cálculos originados en enfermos tratados con indinavir, así como de las cristalurias. Sutherland y cols. ${ }^{1}$ estudiaron por microscopía con luz polarizada un cálculo procedente de un paciente tratado con indinavir. Este estudio reveló que el cálculo no estaba formado por ninguno de los componentes comúnmente asociados a cálculos renales, tales como oxalato cálcico, fosfato cálcico, ácido úrico o cistina. El estudio por medio de espectroscopía infrarroja confirmó este hecho, sugiriendo que el cálculo estaba constituido posiblemente por la precipitación de la droga. 
Estos autores muestran los caracteres morfológicos del cálculo, pero no el espectro originado por el cálculo. Bruce y cols. ${ }^{2}$ analizaron el cálculo procedente de enfermos tratados con indinavir por cromatografía de gas y espectrometría de masas y encontraron que el espectro producido era idéntico al del indinavir puro. Estos autores muestran que los pacientes que interrumpen el tratamiento con indinavir presentan hipocitraturia, un importante inhibidor de la formación de cálculos renales. Es más, el indinavir cristalizado o sus metabolitos podían actuar de núcleo de nucleación heterogénica conduciendo a la formación de otros tipos de cálculos.

Kopp y cols. ${ }^{3}$ utilizaron para el estudio de la identificación de los cálculos renales por indinavir la cromatografia líquida de alta resolución (HPLC) comparando su espectro con el espectro del indinavir puro. Estos autores también emplearon la espectrometría de masas. Trainor y cols. ${ }^{9}$ analizaron los cálculos por espectrofotometría infrarroja.

Las cristalurias procedentes de pacientes tratados con indinavir fueron estudiadas por diversos autores por microscopía con luz polariza$\mathrm{da}^{2,3,9} \mathrm{y}$ también por espectrometría infrarroja.

Nosotros hemos utilizado para el estudio de las cristalurias microscopía con luz polarizada y espectrofotometría infrarroja. El espectro obtenido del indinavir puro, así como el del cálculo y la cristaluria no es coincidente con el espectro del cálculo de indinavir presentado por Trainor ${ }^{9}$. Por otra parte, no hemos encontrado diferencias en los espectros de infrarrojo tanto de la cristaluria como del cálculo al ser sometidos a $70^{\circ} \mathrm{C}$ durante 4 horas. La cristaluria presentó los mismos caracteres que en estudios de otros autores ${ }^{3,9}$.

En todos los pacientes que tomaron indinavir hemos encontrado al igual que Berns ${ }^{11} \mathrm{y} \mathrm{Kopp}^{3} \mathrm{el}$ característico color amarillo que contiene la orina de los pacientes. Es importante destacar que de los 9 pacientes tratados con indinavir, 2 padecieron cólico renal y presentaron abundante cristaluria y uno de ellos además desarrolló un cálculo renal. El resto de los pacientes no presentaron esta sintomatología. La metabolización de los inhibidores de proteasas del VIH-1 y/o SIDA se realiza en el hígado ${ }^{4,8}$ a través del sistema enzimático citocromo $\mathrm{P} 450$, por ello este hecho es de interés porque los enfermos que padecen altera- ciones hepáticas y son tratados con indinavir incrementan la concentración de indinavir en el suero. Dado el alto riesgo de desarrollo de nefrolitiasis en los pacientes que reciben indinavir es de interés tomar las medidas profilácticas adecuadas, como es un alto grado de hidratación.

\section{REFERENCIAS}

1. SUTHERLAND SE, REIGLE MD, SEFTEL AD, RESNICK MI.: Protease inhibitors and urolithiasis. J Urology 1997; 158: 3133.

2. BRUCE RE, MUNCH LC, HOVEN AD, JERAULD RS, GREENBURG RS, PORTER WH et al.: Urolithiasis associated with the protease inhibitor indinavir. Urology 1997; 50: 513-518.

3. KOPP JB, MILLER KD, MICAN JAM FEUERSTEIN IM, VAUGHAN E, BAKER C et al.: Crystalluria and urinary tract abnormalities associated. Ann Intern Med 1997; 127: 119125.

4. GRABE DW, EISELE G, MILLER C SINGH J, STEIN D.: Indinavir-induced nephropathy. Clin Nephrol 1999; 51: 181183.

5. PERAZELLA MA, KASHGARIAN M, COONEY E.: Indinavir nephropathy in an AIDS patient with renal insufficiency and pyuria. Clin Nephrol 1998; 50: 194-196.

6. DIELEMAN JP, STURKENBOOM MC, JAMBROES M, GYSSENS IC, WEVERLING GJ, TEN VEEN JH et al.: Risk factors for urological symptoms in a cohort of users of the HIV protease inhibitor indinavir sulphate. Arch Intern Med 2002; 162: 1493-1501.

7. GENTLE DL, STOLLER ML, JARRETT TW, WARD JF, GEIB KS, WOOD AF et al.: Protease inhibitor-induced urolithiasis. Urology 1997; 50: 508-511.

8. KAKUDA TN, STRUBLE KA, PISCITELLI SC.: Protease inhibitors for the treatment of human immunodeficiency virus infection. Am J Health Syst Pharm 1998; 55: 233-254.

9. TRAINOR LD, STEINBERG JP, AUSTIN GW, SOLOMON HM. Indinavir crystalluria. Arch Pathol Lab Med 1998; 122: 256259.

10. SALTEL E, ANGEL JB, FUTTER NG, WALSH WG, O'ROURKE $\mathrm{K}, \mathrm{MAHONEY} \mathrm{JE}$ et al.: Increased prevalence and analysis of risk factors for indinavir nephrolithiasis. J Urology 2000; 164: 1895-1897.

11. BERNS JS, COHEM RM, SILVERMAN M.: Acute renal failure due to indinavir crystalluria and nephrolithiasis: report of two cases. Am J Kidney Diseases 1997; 30: 558-560.

12. YEH KC, DEUTSCH PJ, HADDIX H, HESNEY M, HOAGLAND V, JU WD et al.: Single-dose pharmacokinetics of indinavir and the effect of food. Am Society Microbiology 1998; 42: 332-338.

13. RACE EM, ADELSON-MITTY J, KRIEGEL GR, BARLAM TF, REIMANN KA, LETVIN NL et al.: Focal mycobacterial lymphodenitis following initiation of protease-inhibitor therapy in patient with advanced HIV-1 disease. Lancet 1998; 351: 252255.

14. RAPADO A, TRABA ML, CAYCHO C, CIFUENTES-DELATTE L.: Drug-induced renal stones: incidence, clinical expression and stone analysis. Contr Nephrol 1987; 58: 25-29.

Dra. $M^{a}$.L. Traba Villameytide

Laboratorio Bioquímica. Fundación Jiménez Díaz

Avda. Reyes Católicos, 2

28040 Madrid

(Trabajo recibido el 5 marzo de 2004) 